\title{
Polyphenols and Food Quality
}

\author{
Raúl Ferrer-Gallego, ${ }^{1}$ Rosa Pérez-Gregorio, ${ }^{2}$ José M. Hernández-Hierro, $^{3}$ \\ Natalia Quijada-Morín, ${ }^{4}$ and Ignacio García-Estévez ${ }^{5}$ \\ ${ }^{1}$ VITEC (Wine Technology Centre), Falset, 43730 Tarragona, Spain \\ ${ }^{2}$ LAQV-REQUIMTE, Departamento de Química e Bioquímica, Faculdade de Ciências, Universidade do Porto, \\ 4169-007 Porto, Portugal \\ ${ }^{3}$ Food Colour and Quality Laboratory, Á. Nutrición y Bromatología, Facultad de Farmacia, Universidad de Sevilla, \\ 41012 Sevilla, Spain \\ ${ }^{4}$ UMR 1083 INRA Sciences pour Enologie, Faculté de Pharmacie, Université de Montpellier, 34000 Montpellier, France \\ ${ }^{5}$ Grupo de Investigación en Polifenoles, Unidad de Nutrición y Bromatología, Facultad de Farmacia, University of Salamanca, \\ 37007 Salamanca, Spain
}

Correspondence should be addressed to Raúl Ferrer-Gallego; raul.ferrer@vitec.cat

Received 12 June 2017; Accepted 12 June 2017; Published 16 July 2017

Copyright (C) 2017 Raúl Ferrer-Gallego et al. This is an open access article distributed under the Creative Commons Attribution License, which permits unrestricted use, distribution, and reproduction in any medium, provided the original work is properly cited.

Polyphenols are abundant secondary metabolites present in many species of plants. Over the lasts years, polyphenols have shown increasingly interest in Food Science. The intake of polyphenols in our diet is closely related not only to human health but also to food quality, since these compounds are greatly related to organoleptic properties and biological activity. This special issue addressed novel research regarding the importance of polyphenols for food quality from different point of view from their biological activity and their importance in nutrition to their role in the quality and the organoleptic properties of food.

Among the submitted works, five papers have been selected to be part of this special issue. The paper authored by Y. Zhao et al. is focused on the potential use of some polyphenols as food preservatives. These authors have carried out comparative studies on the effects of several food preservatives (among them, tea catechin) on bacteria growth. The aim of this work was to assess these products for use as antimicrobial agents in food preservation avoiding the bacteria proliferation (Staphylococcus aureus) and the secretion of enterotoxins that have been associated with food poisoning. The results obtained in this study indicated that tea catechin not only suppressed $S$. aureus growth but also inhibited Staphylococcal Enterotoxin I production and secretion. This work reported that the natural preservatives exert higher antibacterial activity than the chemical ones, such as sodium nitrite, polylysine, or chitosan, and that tea catechin was the most efficient among food preservatives assayed for keeping the food from the contamination with enterotoxins. These investigations would be useful for food industry to provide safer food products due to $S$. aureus enterotoxins-related control strategy.

J. O. Jiménez-Zurita et al. have carried out studies on the effect of different postharvest cold storage on the phenolic composition and physicochemical and biochemical properties of soursop fruit. These studies were mainly focused on the fruit weight and firmness changes and on their composition and enzymatic activity depending on the storage employed during postharvest maturation of soursop fruit. The reported results pointed out that the concentrations of phenolic compounds decreased by the end of the ripening. The highest levels of phenolic compounds were reached when fruits were stored at $22^{\circ} \mathrm{C}$. However, storage at $15^{\circ} \mathrm{C}$ did not affect the biochemical processes related to ripening. Indeed, the occurrence of chilling injury in fruits was avoided. Hence, the shelf life of the fruit increases conserving the organoleptic properties of ripened fruit. 
With regard to the biological activity of food polyphenols, F. Gai et al. have studied the changes in the total polyphenolics and antioxidative capacity of the perilla (Perilla frutescens L.) plant, during the growth cycle. This plant, which has traditionally been used as a source of human and animal food mostly in some Asian countries, such as Korea, Japan, and China, has been reported to be rich in fat and good quality proteins. Moreover, in this paper, authors reported the phenolic composition of this plant at five different growth stages and they correlated it with the antioxidant activity. The phenolic compound profile was characterized by the presence of three major compounds, with rosmarinic acid being the most abundant one. Moreover, the phenolic contents and the antioxidant activity were significantly different according to the growth stage. These data may be useful in determining the optimal harvest time at which phenolic compounds reach their maximum content, which, in turn, are related to a higher antioxidant activity. The results obtained allowed the authors to point out that perilla may constitute a new functional food and could be an important dietary source for the prevention of diseases caused by oxidative stress.

This special issue also includes the study performed by I. Jerez-Martel et al. in which the phenolic composition and the antioxidant activity of crude extracts from both microalgae and cyanobacteria strains have been studied. The seaweed extracts have been proposed as additives for food enrichment due to their preservative properties and/or nutritional benefits. Thus, different extracts of several microalgae and cyanobacteria were studied for their antioxidant activity and phenolic contents. The results obtained by these authors highlight the effectiveness of microalgae and cyanobacteria extracts as scavengers of free radicals and that this activity might be related to the phenolics compounds that were detected. In particular, high contents of phenolic compounds such as gallic acid, protocatechuic acid, (+) catechin, chlorogenic acid, and (-) epicatechin were found in the extract that showed the highest antioxidant capacity (which was obtained from microalgae Euglena cantabrica). These findings could be relevant in order to consider the microalgae and cyanobacteria extracts to be used to different industrial applications during food processing.

The study carried out by E. M. Tanvir et al. is focused on the antioxidant properties of different varieties of turmeric (Curcuma longa) from Bangladesh. Turmeric is a golden spice derived from Curcuma longa plant, which has been traditionally used not only as principal ingredient of some Bangladesh and India dishes but also in folk medicines against various ailments and infectious diseases. The results reported strongly suggest that the turmeric varieties from Bangladesh, in particular "mura" turmeric variety from Chittagong, are promising sources of natural antioxidants, which can be related to their high contents on polyphenols, flavonoids, tannins, and ascorbic acid. Moreover this work reported that the extraction of antioxidant compounds is more effective by using ethanol. Hence, this study suggests the importance of the turmeric varieties investigated as sources of natural antioxidants.
We are pleased to present this special issue, which includes five papers that provide new insight into the interesting field of the relationship between polyphenols and food quality and we sincerely hope that the readers will find this special issue interesting and informative.

\section{Acknowledgments}

Finally, we would like to acknowledge the authors of the papers for their contributions as well as all the reviewers for their valuable participation in the evaluation process.

\author{
Raúl Ferrer-Gallego \\ Rosa Pérez-Gregorio \\ José M. Hernández-Hierro \\ Natalia Quijada-Morín \\ Ignacio García-Estévez
}



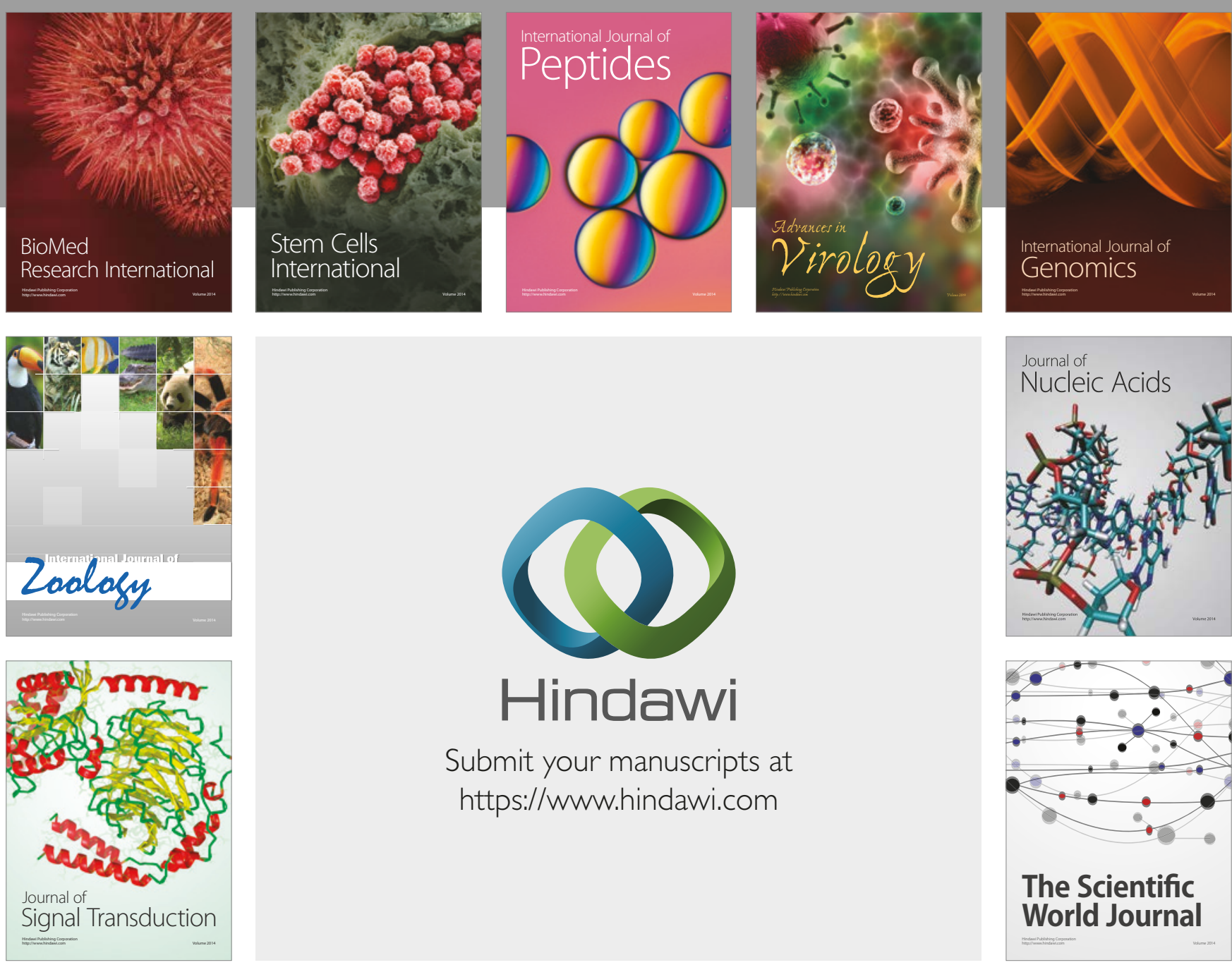

Submit your manuscripts at

https://www.hindawi.com
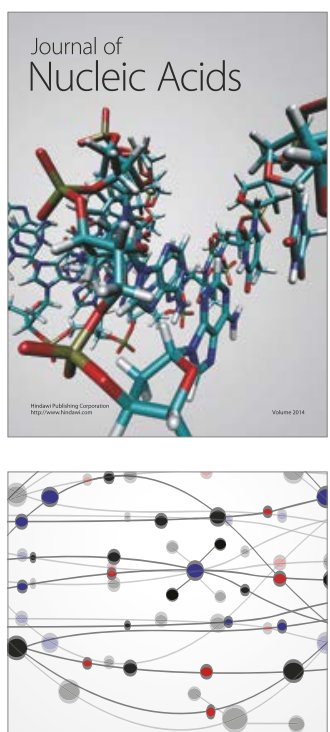

The Scientific World Journal

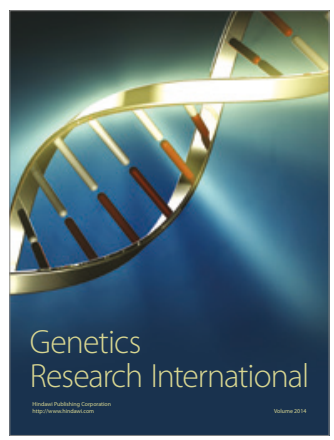

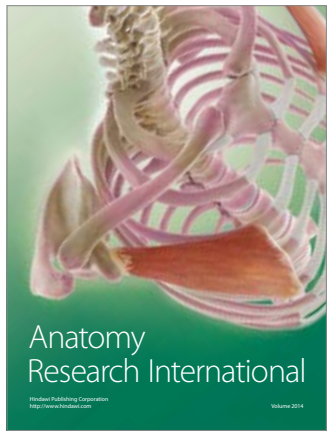

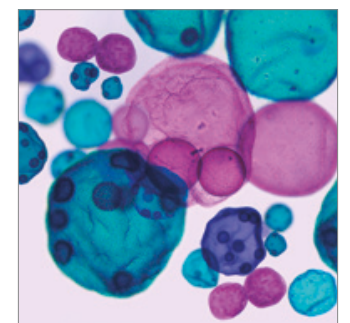

International Journal of Microbiology
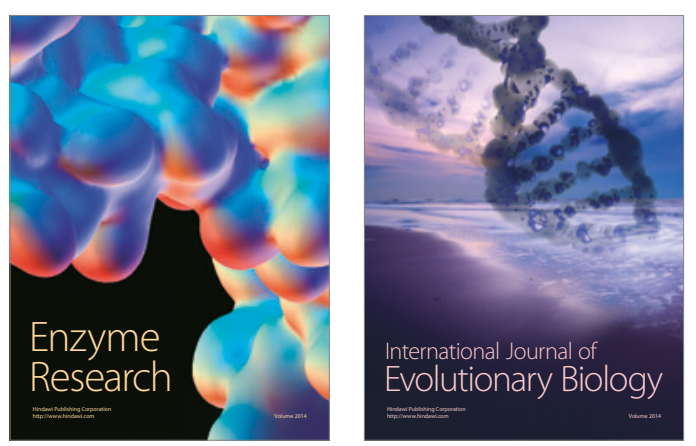
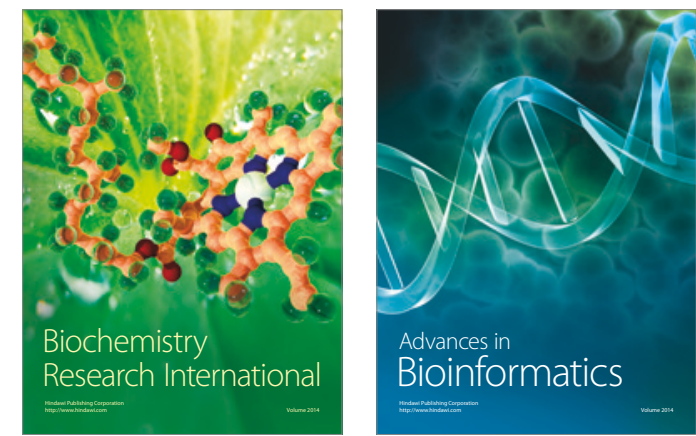

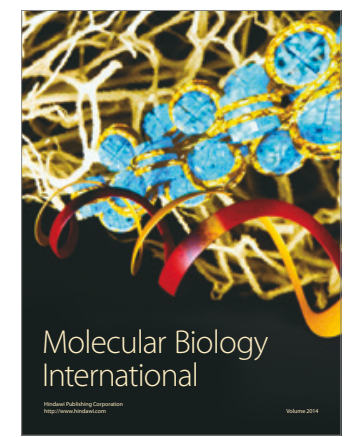

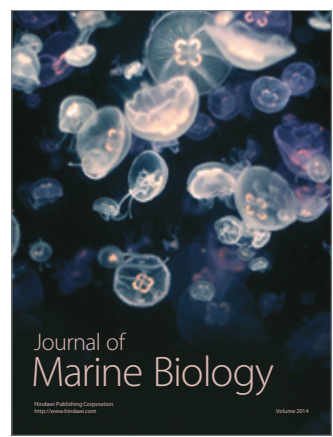

